\title{
Potential Symmetries, One-Dimensional Optimal System and Invariant Solutions of the Coupled Burgers' Equations
}

\author{
Yuexing Bai1,2, Sudao Bilige ${ }^{1 *}$, Temuer Chaolu ${ }^{2}$ \\ ${ }^{1}$ College of Sciences, Inner Mongolia University of Technology, Hohhot, China \\ ${ }^{2}$ College of Arts and Sciences, Shanghai Maritime University, Shanghai, China \\ Email: *inmathematica@126.com
}

How to cite this paper: Bai, Y.X., Bilige, S. and Chaolu, T. (2018) Potential Symmetries, One-Dimensional Optimal System and Invariant Solutions of the Coupled Burgers' Equations. Journal of Applied Mathematics and Physics, 6, 1825-1839. https://doi.org/10.4236/jamp.2018.69156

Received: August 9, 2018

Accepted: September 8, 201

Published: September 11, 2018

Copyright $\odot 2018$ by authors and Scientific Research Publishing Inc. This work is licensed under the Creative Commons Attribution International License (CC BY 4.0).

http://creativecommons.org/licenses/by/4.0/

\begin{abstract}
In this paper, we discuss one-dimensional optimal system and the invariant solutions of Coupled Burgers' equations. By using Wu-differential characteristic set algorithm with the aid of Mathematica software, the classical symmetries of the Coupled Burgers' equations are calculated, and the one-dimensional optimal system of Lie algebra is constructed. And we obtain the invariant solution of the Coupled Burgers' equations corresponding to one element in one dimensional optimal system by using the invariant method. The results generalize the exact solutions of the Coupled Burgers' equations.
\end{abstract}

\section{Keywords}

Potential Symmetry, One-Dimensional Optimal System, Invariant Solution, Coupled Burgers' Equations

\section{Introduction}

Lie group methods are perhaps the most powerful currently available in finding exact solutions of nonlinear partial differential equations (PDEs) [1]. This method has a profound impact on both pure and applied areas of mathematics, physics and mechanics, etc. [2] [3] [4]. Based on the symmetries of a PDEs, many important properties of the equation such as Lie algebras [5], conservation laws [6] [7] [8], exact solutions [9] [10] [11], boundary value problem [12] can be considered successively.

In 1988, Bluman and Kumei [13] suggested a method to find a new class of symmetry for PDEs which can be written as conservative form. They analyzed 
the Lie symmetries of the system $S$ that is obtained by introducing a potential as a further unknown function. In general, not all equations have potential symmetry, because the determining equations (DTEs) of potential symmetry are less than the DTEs of Lie symmetry. Potential symmetry can generate new symmetry and new invariant solutions which could not be obtained by Lie symmetry. Therefore, the study of potential symmetry of PDEs has become an important research topic in many fields [14]. Researchers such as Qu Changzheng [15] and Sophocleous [16] have made outstanding contributions in this field.

The plan of the paper is organized as follows. In Section 2, we obtained the potential symmetries and the one-dimensional optimal system of the Coupled Burgers' equations based on differential characteristic set algorithm, and the corresponding Lie transformation groups are derived. In Section 3, we gave some new invariant solutions and exact solutions of the Coupled Burgers' equations by applying Lie transformation groups on the invariant solutions. In Section 4, we give some discussions and conclusion remarks.

\section{The Potential Symmetries, One-Dimensional Optimal System and Lie Transformation Groups of Coupled Burgers' Equations}

Let's consider Coupled Burgers' equations of Ref. [17],

$$
\begin{aligned}
& u_{t}-u_{x x}+2 u u_{x}+\alpha(u v)_{x}=0, \\
& v_{t}-v_{x x}+2 v v_{x}+\beta(u v)_{x}=0 .
\end{aligned}
$$

where $\alpha, \beta$ are constants. The conserved form of Equations (1) (2) have the following forms

$$
\begin{aligned}
& u_{t}-\left[u_{x}-u^{2}-\alpha(u v)\right]_{x}=0, \\
& v_{t}-\left[v_{x}-v^{2}-\beta(u v)\right]_{x}=0 .
\end{aligned}
$$

For obtaining the potential symmetry of Coupled Burgers' equations, we introduce the potential variables $\phi, \psi$, then Equations (3) (4) are equivalent to the potential systems

$$
\left\{\begin{array}{l}
\phi_{x}=u, \\
\phi_{t}=u_{x}-u^{2}-\alpha(u v), \\
\psi_{x}=v, \\
\psi_{t}=v_{x}-v^{2}-\beta(u v) .
\end{array}\right.
$$

The symmetry group of Equations (5) will be generated by the vector field of the form

$$
\begin{aligned}
X= & \xi(x, t, u, v, \phi, \psi) \frac{\partial}{\partial x}+\tau(x, t, u, v, \phi, \psi) \frac{\partial}{\partial t}+\eta_{1}(x, t, u, v, \phi, \psi) \frac{\partial}{\partial u} \\
& +\eta_{2}(x, t, u, v, \phi, \psi) \frac{\partial}{\partial v}+\gamma(x, t, u, v, \phi, \psi) \frac{\partial}{\partial \varphi}+\delta(x, t, u, v, \phi, \psi) \frac{\partial}{\partial \psi}
\end{aligned}
$$


where $\xi=\xi(x, t, u, v, \phi, \psi), \quad \tau=\tau(x, t, u, v, \phi, \psi), \quad \eta_{1}=\eta_{1}(x, t, u, v, \phi, \psi)$, $\eta_{2}=\eta_{2}(x, t, u, v, \phi, \psi), \gamma=\gamma(x, t, u, v, \phi, \psi), \delta=\delta(x, t, u, v, \phi, \psi)$ are the infinitesimal functions.

Definition 1. If $\xi_{\phi}^{2}+\tau_{\phi}^{2}+\eta_{1 \phi}^{2}+\eta_{2 \phi}^{2} \neq 0, \quad \xi_{\psi}^{2}+\tau_{\psi}^{2}+\eta_{1 \psi}^{2}+\eta_{2 \psi}^{2} \neq 0$, if and only if the infinitesimal functions $\xi, \tau, \eta_{1}, \eta_{2}$ have an essential dependence on the potential variables $\phi, \psi$, then $X$ is called the potential symmetry vector of Coupled Burgers' Equations (1) (2).

\subsection{The Potential Symmetries}

In this section, we can divide it into three cases as following.

Case 1: $\alpha \neq 0, \beta \neq 0$.

According to the Lie algorithm, we obtain the determining equations of symmetry (6), but it is too difficult to get its solutions. However, we can obtain the following equivalent system of the determining system by corresponding to the characteristic set which is equivalent to the determining equations by using the differential characteristic set algorithm of Ref. [18]

$$
\begin{gathered}
2 \eta_{1}+u \tau_{t}=0, \tau_{x}=\tau_{u}=\tau_{v}=\tau_{\phi}=\tau_{\psi}=0, \eta_{1 x}=\eta_{1 t}=\eta_{1 v}=\eta_{1 \phi}=\eta_{1 \psi}=0, \\
\gamma_{x}=\gamma_{t}=\gamma_{u}=\gamma_{v}=\gamma_{\phi}=\gamma_{\psi}=0, \delta_{x}=\delta_{t}=\delta_{u}=\delta_{v}=\delta_{\phi}=\delta_{\psi}=0, \\
\xi_{t}=\xi_{u}=\xi_{v}=\xi_{\phi}=\xi_{\psi}=0, \eta_{1}+u \eta_{1 u}=0, \eta_{1}+u \xi_{x}=0, v \eta_{1}-u \eta_{2}=0 .
\end{gathered}
$$

By solving above equations, we obtain the infinitesimal functions as follows:

$$
\xi=-c_{3} x+c_{5}, \tau=-2 c_{3} t+c_{4}, \eta_{1}=c_{3} u, \eta_{2}=c_{3} v, \gamma=c_{1}, \delta=c_{2},
$$

where $c_{i}(i=1, \cdots, 5)$ are arbitrary symmetry parameters, and can give five finite symmetries of Equations (1) (2):

$$
X_{1}=\frac{\partial}{\partial \phi}, X_{2}=\frac{\partial}{\partial \psi}, X_{3}=-x \frac{\partial}{\partial x}-2 t \frac{\partial}{\partial t}+u \frac{\partial}{\partial u}+v \frac{\partial}{\partial v}, X_{4}=\frac{\partial}{\partial t}, X_{5}=\frac{\partial}{\partial x} .
$$

According to the definition 1, they are not potential symmetries.

Case 2: $\alpha=0, \beta \neq 0$, Equations (1) (2) have the following forms:

$$
\begin{gathered}
u_{t}-u_{x x}+2 u u_{x}=0, \\
v_{t}-v_{x x}+2 v v_{x}+\beta(u v)_{x}=0,
\end{gathered}
$$

and Equations (5) have the following forms

$$
\left\{\begin{array}{l}
\phi_{x}=u, \\
\phi_{t}=u_{x}-u^{2}, \\
\psi_{x}=v, \\
\psi_{t}=v_{x}-v^{2}-\beta(u v) .
\end{array}\right.
$$

By the same manner, we can obtain the following equations:

$$
\begin{gathered}
\xi_{t}=\xi_{u}=\xi_{v}=\xi_{\phi}=\xi_{\psi}=0, \tau_{x}=\tau_{u}=\tau_{v}=\tau_{\phi}=\tau_{\psi}=0, \\
\eta_{1 x}=\eta_{1 t}=\eta_{1 v}=\eta_{1 \phi}=\eta_{1 \psi}=0, \eta_{2 x}=\eta_{2 t}=\eta_{2 u u}=0, \\
\gamma_{x}=\gamma_{t}=\gamma_{u}=\gamma_{v}=\gamma_{\phi}=\gamma_{\psi}=0, \delta_{x}=\delta_{t}=\delta_{u}=\delta_{v}=0, \\
\eta_{1}-u \eta_{1 u}=0,2 \eta_{1}+u \tau_{t}=0, \eta_{1}+u \xi_{x}=0, v \eta_{1}-u \eta_{2}+u \eta_{2 \psi}=0,
\end{gathered}
$$




$$
\begin{gathered}
\eta_{2}-v \eta_{2 v}-u \eta_{2 u}=0, \eta_{2 \phi}+u \eta_{2 u}-\beta u \eta_{2 u}-v \eta_{2 u}=0, \\
\delta_{\phi}-\eta_{2 u}=0, u v \delta_{\psi}+v \eta_{1}-u \eta_{2}+u^{2} \eta_{2 u}=0 .
\end{gathered}
$$

By calculating above equations, we obtain the infinitesimal functions:

$$
\begin{gathered}
\xi=-c_{2} x+c_{4}, \tau=-2 c_{2} t+c_{3}, \eta_{1}=c_{2} u, \\
\eta_{2}=c_{5}\left(u+\frac{v}{-1+\beta}\right) \mathrm{e}^{\psi+(-1+\beta) \phi}+c_{2} v+c_{6} v \mathrm{e}^{\psi}, \\
\gamma=c_{1}, \delta=\frac{c_{5} \mathrm{e}^{\psi+(-1+\beta) \phi}}{-1+\beta}+c_{6} \mathrm{e}^{\psi}+c_{7},
\end{gathered}
$$

where $c_{i}(i=1, \cdots, 7)$ are arbitrary symmetry parameters, and can give seven finite symmetries of Equations (7) (8):

$$
\begin{gathered}
X_{1}=\frac{\partial}{\partial \phi}, X_{2}=-x \frac{\partial}{\partial x}-2 t \frac{\partial}{\partial t}+u \frac{\partial}{\partial u}+v \frac{\partial}{\partial v}, X_{3}=\frac{\partial}{\partial t}, X_{4}=\frac{\partial}{\partial x}, \\
X_{5}=\left(u+\frac{v}{-1+\beta}\right) \mathrm{e}^{\psi+(-1+\beta) \phi} \frac{\partial}{\partial v}+\frac{\mathrm{e}^{\psi+(-1+\beta) \phi}}{-1+\beta} \frac{\partial}{\partial \psi}, \\
X_{6}=v \mathrm{e}^{\psi /} \frac{\partial}{\partial v}+\mathrm{e}^{\psi} \frac{\partial}{\partial \psi}, X_{7}=\frac{\partial}{\partial \psi} .
\end{gathered}
$$

According to Definition 1, $X_{5}$ is the potential symmetry of Equations (7) (8).

Case 3: $\alpha \neq 0, \beta=0$, Equations (1) (2) have the following forms:

$$
\begin{gathered}
u_{t}-u_{x x}+2 u u_{x}+\alpha(u v)_{x}=0, \\
v_{t}-v_{x x}+2 v v_{x}=0 .
\end{gathered}
$$

and Equations (5) has the following forms

$$
\left\{\begin{array}{l}
\phi_{x}=u, \\
\phi_{t}=u_{x}-u^{2}-\alpha(u v), \\
\psi_{x}=v, \\
\psi_{t}=v_{x}-v^{2}
\end{array}\right.
$$

By the same manner, we can obtain the following equations:

$$
\begin{gathered}
\xi_{t}=\xi_{u}=\xi_{v}=\xi_{\phi}=\xi_{\psi}=0, \tau_{x}=\tau_{u}=\tau_{v}=\tau_{\phi}=\tau_{\psi}=0, \\
\eta_{2 x}=\eta_{2 t}=\eta_{2 u}=\eta_{2 \phi}=\eta_{2 \psi}=0, \eta_{1 x}=\eta_{1 t}=\eta_{1 u u}=0, \\
\gamma_{x}=\gamma_{t}=\gamma_{u}=\gamma_{v}=0, \delta_{x}=\delta_{t}=\delta_{u}=\delta_{v}=\delta_{\phi}=\delta_{\psi}=0, \\
\eta_{2}-v \eta_{2 v}=0,2 \eta_{2}+v \tau_{t}=0, \eta_{2}+v \xi_{x}=0, \\
\eta_{1}-v \eta_{1 v}-u \eta_{1 u}=0, v \eta_{1}-v \eta_{1 \phi}-u \eta_{2}=0, \\
-u \eta_{1}+v \eta_{1}-\alpha v \eta_{1}+v \eta_{1 \psi}+u^{2} \eta_{1 u}-u v \eta_{1 u}+\alpha u v \eta_{1 u}=0, \\
v \gamma_{\psi}-\eta_{1}+u \eta_{1 u}=0, v \gamma_{\phi}-v \eta_{1 u}+\eta_{2}=0 .
\end{gathered}
$$

By calculating above equations, we obtain the infinitesimal functions:

$$
\xi=-c_{2} x+c_{3}, \tau=-2 c_{2} t+c_{4}, \eta_{1}=c_{5}\left(v+\frac{u}{-1+\alpha}\right) \mathrm{e}^{\phi+(-1+\alpha) \psi}+c_{2} u+c_{6} v \mathrm{e}^{\phi},
$$




$$
\eta_{2}=c_{2} v, \delta=c_{1}, \gamma=\frac{c_{5} \mathrm{e}^{\phi+(-1+\alpha) \psi}}{-1+\alpha}+c_{6} \mathrm{e}^{\phi}+c_{7},
$$

where $c_{i}(i=1, \cdots, 7)$ are arbitrary symmetry parameters, and can give seven finite symmetries of Equations (10) (11):

$$
\begin{gathered}
X_{1}=\frac{\partial}{\partial \psi}, X_{2}=-x \frac{\partial}{\partial x}-2 t \frac{\partial}{\partial t}+u \frac{\partial}{\partial u}+v \frac{\partial}{\partial v}, X_{3}=\frac{\partial}{\partial x}, X_{4}=\frac{\partial}{\partial t} \\
X_{5}=\left(v+\frac{u}{-1+\alpha}\right) \mathrm{e}^{\phi+(-1+\alpha) \psi} \frac{\partial}{\partial u}+\frac{\mathrm{e}^{\phi+(-1+\alpha) \psi}}{-1+\alpha} \frac{\partial}{\partial \phi}, X_{6}=u \mathrm{e}^{\phi} \frac{\partial}{\partial u}+\mathrm{e}^{\phi} \frac{\partial}{\partial \phi}, X_{7}=\frac{\partial}{\partial \phi} .
\end{gathered}
$$

According to Definition 1, $X_{5}$ is the potential symmetry of Equations (10) (11).

\subsection{One-Dimensional Optimal System of the Coupled Burgers' Equations}

As is mentioned in process of Ref. [3], the problem of finding an optimal of subgroups is equivalent to that of finding an optimal system of subalgebras. In this section, we will construct the optimal system of one-dimensional subalgebras of Coupled Burgers' equations Equations (7) (8) by using the method presented in process of Ref. [3].

In the case 2, we have obtained seven operators $X_{1} \sim X_{7}$. Applying the commutator operators $\left[X_{m}, X_{n}\right]=X_{m} X_{n}-X_{n} X_{m}$, we obtain the commutator table listed in Table 1 with the $(i, j)$-th entry indicating $\left[X_{i}, X_{j}\right]$. The commutation relations between these vector fields are given by the following table, the entry in row $i$ and column $j$ representing $\left[X_{i}, X_{j}\right]$.

From the above commutator table for this algebra, we use the Lie series and apply the following formula

$$
\begin{aligned}
\operatorname{Ad}\left(\exp \left(\varepsilon X_{i}\right)\right) X_{j} & =\sum_{n=0}^{\infty} \frac{\varepsilon^{n}}{n !}\left(\operatorname{adX} X_{i}\right)^{n}\left(X_{j}\right) \\
& =X_{j}-\varepsilon\left[X_{i}, X_{j}\right]+\frac{\varepsilon^{2}}{2}\left[X_{i},\left[X_{i}, X_{j}\right]\right]-\cdots
\end{aligned}
$$

and compute the adjoint representation, we obtain the following table with the $(i, j)$-th entry indicating $\operatorname{Ad}\left(\exp \left(\varepsilon X_{i}\right)\right) X_{j}$.

Table 1. Commutator title.

\begin{tabular}{cccccccc}
\hline & $X_{1}$ & $X_{2}$ & $X_{3}$ & $X_{4}$ & $X_{5}$ & $X_{6}$ & $X_{7}$ \\
\hline$X_{1}$ & 0 & 0 & 0 & 0 & $-(-1+\beta) X_{5}$ & 0 & 0 \\
$X_{2}$ & 0 & 0 & $2 X_{3}$ & $X_{4}$ & 0 & 0 & 0 \\
$X_{3}$ & 0 & $-2 X_{3}$ & 0 & 0 & 0 & 0 & 0 \\
$X_{4}$ & 0 & $-X_{4}$ & 0 & 0 & 0 & 0 & 0 \\
$X_{5}$ & $-(-1+\beta) X_{5}$ & 0 & 0 & 0 & 0 & 0 & $-X_{5}$ \\
$X_{6}$ & 0 & 0 & 0 & 0 & 0 & 0 & $-X_{6}$ \\
$X_{7}$ & 0 & 0 & 0 & 0 & $X_{5}$ & $X_{6}$ & 0 \\
\hline
\end{tabular}


With the help of the adjoint representation Table 2, applying the adjoint action of $X$ to $X=a_{1} X_{1}+a_{2} X_{2}+a_{3} X_{3}+a_{4} X_{4}+a_{5} X_{5}+a_{6} X_{6}+a_{7} X_{7}$, we have

$$
\begin{aligned}
& \operatorname{Ad}\left(\exp \left(\varepsilon_{1} X_{1}\right)\right) X \\
& =a_{1} X_{1}+a_{2} X_{2}+a_{3} X_{3}+a_{4} X_{4}+a_{5} \mathrm{e}^{-\varepsilon_{1}(-1+\beta)} X_{5}+a_{6} X_{6}+a_{7} X_{7} \\
& =\left(X_{1}, X_{2}, X_{3}, X_{4}, X_{5}, X_{6}, X_{7}\right) \cdot \boldsymbol{Q}_{1} \cdot\left(a_{1}, a_{2}, a_{3}, a_{4}, a_{5}, a_{6}, a_{7}\right)^{\mathrm{T}} .
\end{aligned}
$$

It is easy to obtain $\boldsymbol{Q}_{1}$ as follow,

$$
\boldsymbol{Q}_{1}=\left(\begin{array}{ccccccc}
1 & 0 & 0 & 0 & 0 & 0 & 0 \\
0 & 1 & 0 & 0 & 0 & 0 & 0 \\
0 & 0 & 1 & 0 & 0 & 0 & 0 \\
0 & 0 & 0 & 1 & 0 & 0 & 0 \\
0 & 0 & 0 & 0 & \mathrm{e}^{-\varepsilon_{1}(-1+\beta)} & 0 & 0 \\
0 & 0 & 0 & 0 & 0 & 1 & 0 \\
0 & 0 & 0 & 0 & 0 & 0 & 1
\end{array}\right)
$$

Similarly, the other matrices of the separate adjoint actions of the vectors $\boldsymbol{Q}_{2}$, $\boldsymbol{Q}_{3}, \boldsymbol{Q}_{4}, \boldsymbol{Q}_{5}, \boldsymbol{Q}_{6}, \boldsymbol{Q}_{7}$ are found as follows:

$$
\begin{aligned}
& \boldsymbol{Q}_{2}=\left(\begin{array}{ccccccc}
1 & 0 & 0 & 0 & 0 & 0 & 0 \\
0 & 1 & 0 & 0 & 0 & 0 & 0 \\
0 & 0 & \mathrm{e}^{-2 \varepsilon_{2}} & 0 & 0 & 0 & 0 \\
0 & 0 & 0 & \mathrm{e}^{-\varepsilon_{2}} & 0 & 0 & 0 \\
0 & 0 & 0 & 0 & 1 & 0 & 0 \\
0 & 0 & 0 & 0 & 0 & 1 & 0 \\
0 & 0 & 0 & 0 & 0 & 0 & 1
\end{array}\right), \boldsymbol{Q}_{3}=\left(\begin{array}{ccccccc}
1 & 0 & 0 & 0 & 0 & 0 & 0 \\
0 & 1 & 0 & 0 & 0 & 0 & 0 \\
0 & 2 \varepsilon_{3} & 1 & 0 & 0 & 0 & 0 \\
0 & 0 & 0 & 1 & 0 & 0 & 0 \\
0 & 0 & 0 & 0 & 1 & 0 & 0 \\
0 & 0 & 0 & 0 & 0 & 1 & 0 \\
0 & 0 & 0 & 0 & 0 & 0 & 1
\end{array}\right), \\
& \boldsymbol{Q}_{4}=\left(\begin{array}{ccccccc}
1 & 0 & 0 & 0 & 0 & 0 & 0 \\
0 & 1 & 0 & 0 & 0 & 0 & 0 \\
0 & 0 & 1 & 0 & 0 & 0 & 0 \\
0 & \varepsilon_{4} & 0 & 1 & 0 & 0 & 0 \\
0 & 0 & 0 & 0 & 1 & 0 & 0 \\
0 & 0 & 0 & 0 & 0 & 1 & 0 \\
0 & 0 & 0 & 0 & 0 & 0 & 1
\end{array}\right), \boldsymbol{Q}_{5}=\left(\begin{array}{ccccccc}
1 & 0 & 0 & 0 & 0 & 0 & 0 \\
0 & 1 & 0 & 0 & 0 & 0 & 0 \\
0 & 0 & 1 & 0 & 0 & 0 & 0 \\
0 & 0 & 0 & 1 & 0 & 0 & 0 \\
\varepsilon_{5}(-1+\beta) & 0 & 0 & 0 & 1 & 0 & \varepsilon_{5} \\
0 & 0 & 0 & 0 & 0 & 1 & 0 \\
0 & 0 & 0 & 0 & 0 & 0 & 1
\end{array}\right), \\
& \boldsymbol{Q}_{6}=\left(\begin{array}{ccccccc}
1 & 0 & 0 & 0 & 0 & 0 & 0 \\
0 & 1 & 0 & 0 & 0 & 0 & 0 \\
0 & 0 & 1 & 0 & 0 & 0 & 0 \\
0 & 0 & 0 & 1 & 0 & 0 & 0 \\
0 & 0 & 0 & 0 & 1 & 0 & 0 \\
0 & 0 & 0 & 0 & 0 & 1 & \varepsilon_{6} \\
0 & 0 & 0 & 0 & 0 & 0 & 1
\end{array}\right), \boldsymbol{Q}_{7}=\left(\begin{array}{ccccccc}
1 & 0 & 0 & 0 & 0 & 0 & 0 \\
0 & 1 & 0 & 0 & 0 & 0 & 0 \\
0 & 0 & 1 & 0 & 0 & 0 & 0 \\
0 & 0 & 0 & 1 & 0 & 0 & 0 \\
0 & 0 & 0 & 0 & \mathrm{e}^{-\varepsilon_{7}} & 0 & 0 \\
0 & 0 & 0 & 0 & 0 & \mathrm{e}^{-\varepsilon_{7}} & 0 \\
0 & 0 & 0 & 0 & 0 & 0 & 1
\end{array}\right) .
\end{aligned}
$$

Then the general adjoint transformation matrix $\boldsymbol{Q}$ of the Coupled Burgers' equations is

$$
\boldsymbol{Q}=\left(a_{i, j}\right)_{7 \times 7}=\boldsymbol{Q}_{1} \boldsymbol{Q}_{2} \boldsymbol{Q}_{3} \boldsymbol{Q}_{4} \boldsymbol{Q}_{5} \boldsymbol{Q}_{6} \boldsymbol{Q}_{7},
$$


Table 2. Adjoint title.

\begin{tabular}{cccccccc}
\hline Ad & $X_{1}$ & $X_{2}$ & $X_{3}$ & $X_{4}$ & $X_{5}$ & $X_{6}$ & $X_{7}$ \\
\hline$X_{1}$ & $X_{1}$ & $X_{2}$ & $X_{3}$ & $X_{4}$ & $\mathrm{e}^{-(-1+\beta) \varepsilon} X_{5}$ & $X_{6}$ & $X_{7}$ \\
$X_{2}$ & $X_{1}$ & $X_{2}$ & $\mathrm{e}^{-2 \varepsilon} X_{3}$ & $\mathrm{e}^{-\varepsilon} X_{4}$ & $X_{5}$ & $X_{6}$ & $X_{7}$ \\
$X_{3}$ & $X_{1}$ & $X_{2}+2 \varepsilon X_{3}$ & $X_{3}$ & $X_{4}$ & $X_{5}$ & $X_{6}$ & $X_{7}$ \\
$X_{4}$ & $X_{1}$ & $X_{2}+\varepsilon X_{4}$ & $X_{3}$ & $X_{4}$ & $X_{5}$ & $X_{6}$ & $X_{7}$ \\
$X_{5}$ & $X_{1}+\varepsilon(-1+\beta) X_{5}$ & $X_{2}$ & $X_{3}$ & $X_{4}$ & $X_{5}$ & $X_{6}$ & $X_{7}+\mathrm{E} X_{5}$ \\
$X_{6}$ & $X_{1}$ & $X_{2}$ & $X_{3}$ & $X_{4}$ & $X_{5}$ & $X_{6}$ & $X_{7}+\varepsilon X_{6}$ \\
$X_{7}$ & $X_{1}$ & $X_{2}$ & $X_{3}$ & $X_{4}$ & $\mathrm{e}^{-\varepsilon} X_{5}$ & $\mathrm{e}^{-\varepsilon} X_{6}$ & $X_{7}$ \\
\hline
\end{tabular}

the general adjoint transformation equation of the Coupled Burgers' equations is

$$
\frac{1}{a} \boldsymbol{Q}\left(\alpha_{1}, \alpha_{2}, \alpha_{3}, \alpha_{4}, \alpha_{5}, \alpha_{6}, \alpha_{7}\right)^{\mathrm{T}}=\left(\beta_{1}, \beta_{2}, \beta_{3}, \beta_{4}, \beta_{5}, \beta_{6}, \beta_{7}\right)^{\mathrm{T}}
$$

where $a \neq 0$. According to Equation (15), we got

$$
\frac{1}{a}\left(\begin{array}{c}
\alpha_{1} \\
\alpha_{2} \\
\alpha_{3} \mathrm{e}^{-2 \varepsilon_{2}}+2 \alpha_{2} \varepsilon_{3} \mathrm{e}^{-2 \varepsilon_{2}} \\
\mathrm{e}^{-\varepsilon_{2}}\left(\alpha_{4}+\alpha_{2} \varepsilon_{4}\right) \\
\alpha_{5} \mathrm{e}^{(1-\beta) \varepsilon_{1}-\varepsilon_{7}}+\alpha_{7} \varepsilon_{5} \mathrm{e}^{(1-\beta) \varepsilon_{1}}+\alpha_{1} \varepsilon_{5}(-1+\beta) \mathrm{e}^{(1-\beta) \varepsilon_{1}} \\
\alpha_{6} \mathrm{e}^{-\varepsilon_{7}}+\alpha_{7} \varepsilon_{6} \\
\alpha_{7}
\end{array}\right)=\left(\begin{array}{c}
\beta_{1} \\
\beta_{2} \\
\beta_{3} \\
\beta_{4} \\
\beta_{5} \\
\beta_{6} \\
\beta_{7}
\end{array}\right) .
$$

Case 2.1 According to Equation (16), when $\alpha_{7} \neq 0,\left(\alpha_{7}=a, \beta_{7}=1\right)$, it can also be divided into four cases:

1) When $\alpha_{1} \neq 0, \alpha_{2} \neq 0$, let $\beta_{3}=\beta_{4}=\beta_{5}=\beta_{6}=0$, we have $\varepsilon_{3}=-\frac{\alpha_{3}}{2 \alpha_{2}}$, $\varepsilon_{4}=-\frac{\alpha_{4}}{\alpha_{2}}, \quad \varepsilon_{5}=-\frac{\mathrm{e}^{-\varepsilon_{7}} \alpha_{5}}{\alpha_{7}+\alpha_{1}(-1+\beta)}, \quad \varepsilon_{6}=-\frac{\mathrm{e}^{-\varepsilon_{7}} \alpha_{6}}{\alpha_{7}}$ and $\overline{\beta_{1}}=(\lambda, \lambda, 0,0,0,0,1)$.

2) When $\alpha_{1} \neq 0, \alpha_{2}=0$, Equation (16) has the follow formula

$$
\frac{1}{a}\left(\begin{array}{c}
\alpha_{1} \\
0 \\
\alpha_{3} \mathrm{e}^{-2 \varepsilon_{2}} \\
\mathrm{e}^{-\varepsilon_{2}} \alpha_{4} \\
\alpha_{5} \mathrm{e}^{(1-\beta) \varepsilon_{1}-\varepsilon_{7}}+\alpha_{7} \varepsilon_{5} \mathrm{e}^{(1-\beta) \varepsilon_{1}}+\alpha_{1} \varepsilon_{5}(-1+\beta) \mathrm{e}^{(1-\beta) \varepsilon_{1}} \\
\alpha_{6} \mathrm{e}^{-\varepsilon_{7}}+\alpha_{7} \varepsilon_{6} \\
\alpha_{7}
\end{array}\right)=\left(\begin{array}{c}
\beta_{1} \\
\beta_{2} \\
\beta_{3} \\
\beta_{4} \\
\beta_{5} \\
\beta_{6} \\
\beta_{7}
\end{array}\right) .
$$

It can also be divided into four cases:

a) when $\alpha_{3} \neq 0, \alpha_{4} \neq 0$, let $\beta_{5}=\beta_{6}=0$, we have $\varepsilon_{5}=-\frac{\mathrm{e}^{-\varepsilon_{7}} \alpha_{5}}{\alpha_{7}+\alpha_{1}(-1+\beta)}$, $\varepsilon_{6}=-\frac{\mathrm{e}^{-\varepsilon_{7}} \alpha_{6}}{\alpha_{7}}$ and $\overline{\beta_{2}}=(\lambda, 0,1,1,0,0,1)$ 
b) when $\alpha_{3} \neq 0, \alpha_{4}=0$, we can obtain $\beta_{4}=\lambda_{3}$ and $\overline{\beta_{3}}=(\lambda, 0,1,0,0,0,1)$.

c) when $\alpha_{3}=0, \alpha_{4} \neq 0$, we have $\overline{\beta_{4}}=(\lambda, 0,0,1,0,0,1)$.

d) when $\alpha_{3}=0, \alpha_{4}=0$. we have $\overline{\beta_{5}}=(\lambda, 0,0,0,0,0,1)$.

3) When $\alpha_{1}=0, \alpha_{2} \neq 0$, let $\beta_{3}=\beta_{4}=\beta_{5}=\beta_{6}=0$, we have $\varepsilon_{3}=-\frac{\alpha_{3}}{2 \alpha_{2}}$, $\varepsilon_{4}=-\frac{\alpha_{4}}{\alpha_{2}}, \quad \varepsilon_{5}=-\frac{\mathrm{e}^{-\varepsilon_{7}} \alpha_{5}}{\alpha_{7}}, \quad \varepsilon_{6}=-\frac{\mathrm{e}^{-\varepsilon_{7}} \alpha_{6}}{\alpha_{7}}$ and $\overline{\beta_{6}}=(0, \lambda, 0,0,0,0,1)$.

4) When $\alpha_{1}=0, \alpha_{2}=0$, we find that it can also be divided into four cases:

a) If $\alpha_{3} \neq 0, \alpha_{4} \neq 0$, let $\beta_{5}=\beta_{6}=0$, we have $\varepsilon_{5}=-\frac{\mathrm{e}^{-\varepsilon_{7}} \alpha_{5}}{\alpha_{7}}, \varepsilon_{6}=-\frac{\mathrm{e}^{-\varepsilon_{7}} \alpha_{6}}{\alpha_{7}}$ and $\overline{\beta_{7}}=(0,0,1,1,0,0,1)$;

b) If $\alpha_{3} \neq 0, \alpha_{4}=0$, we have $\overline{\beta_{8}}=(0,0,1,0,0,0,1)$;

c) If $\alpha_{3}=0, \alpha_{4} \neq 0$, we have $\overline{\beta_{9}}=(0,0,0,1,0,0,1)$;

d) If $\alpha_{3}=0, \alpha_{4}=0$, we have $\overline{\beta_{10}}=(0,0,0,0,0,0,1)$.

Case 2.2 When $\alpha_{7}=0, \alpha_{6} \neq 0$, we obtain $\beta_{7}=0$. Equation (16) has the follow formula

$$
\frac{1}{a}\left(\begin{array}{c}
\alpha_{1} \\
\alpha_{2} \\
\alpha_{3} \mathrm{e}^{-2 \varepsilon_{2}}+2 \alpha_{2} \varepsilon_{3} \mathrm{e}^{-2 \varepsilon_{2}} \\
\mathrm{e}^{-\varepsilon_{2}}\left(\alpha_{4}+\alpha_{2} \varepsilon_{4}\right) \\
\alpha_{5} \mathrm{e}^{(1-\beta) \varepsilon_{1}-\varepsilon_{7}}+\alpha_{1} \varepsilon_{5}(-1+\beta) \mathrm{e}^{(1-\beta) \varepsilon_{1}} \\
\alpha_{6} \mathrm{e}^{-\varepsilon_{7}} \\
0
\end{array}\right)=\left(\begin{array}{c}
\beta_{1} \\
\beta_{2} \\
\beta_{3} \\
\beta_{4} \\
\beta_{5} \\
\beta_{6} \\
\beta_{7}
\end{array}\right) .
$$

It can also be divided into four cases:

1) When $\alpha_{1} \neq 0, \alpha_{2} \neq 0$, we can obtain $\beta_{2}=\lambda_{7}$, adjust $\frac{1}{a}$, make $\beta_{6}=1$.

Let $\beta_{3}=\beta_{4}=\beta_{5}=0$, we can obtain $\varepsilon_{3}=-\frac{\alpha_{3}}{2 \alpha_{2}}, \varepsilon_{4}=-\frac{\alpha_{4}}{\alpha_{2}}, \varepsilon_{5}=-\frac{\mathrm{e}^{-\varepsilon_{7}} \alpha_{5}}{\alpha_{1}(-1+\beta)}$, we have $\overline{\beta_{11}}=(\lambda, \lambda, 0,0,0,1,0)$.

2) When $\alpha_{1} \neq 0, \alpha_{2}=0$, it can also be divided into four cases:

a) when $\alpha_{3} \neq 0, \quad \alpha_{4} \neq 0$, we have $\overline{\beta_{12}}=(\lambda, 0,1,1,0,1,0)$;

b) when $\alpha_{3} \neq 0, \alpha_{4}=0$, we have $\overline{\beta_{13}}=(1,0,1,0,0,1,0)$;

c) when $\alpha_{3}=0, \alpha_{4} \neq 0$, we have $\overline{\beta_{14}}=(\lambda, 0,0,1,0,1,0)$;

d) when $\alpha_{3}=0, \alpha_{4}=0$, we have $\overline{\beta_{15}}=(1,0,0,0,0,1,0)$.

3) When $\alpha_{1}=0, \alpha_{2} \neq 0$, if $\alpha_{5} \neq 0$,

$$
\mathrm{e}^{(1-\beta) \varepsilon_{1}-\varepsilon_{7}} \alpha_{5}= \begin{cases}1, & \alpha_{5}>0 \\ -1, & \alpha_{5}<0\end{cases}
$$

We have $\overline{\beta_{16}}=(0,1,0,0, \pm 1,1,0)$, if $\alpha_{5}=0$, we have $\overline{\beta_{17}}=(0,1,0,0,0,1,0)$.

4) When $\alpha_{1}=0, \alpha_{2}=0$, we find that it can also be divided into eight cases:

a) when $\alpha_{3} \neq 0, \quad \alpha_{4} \neq 0, \quad \alpha_{5} \neq 0$, we have $\overline{\beta_{18}}=(0,0,1,1, \pm 1,1,0)$;

b) when $\alpha_{3} \neq 0, \alpha_{4}=0, \alpha_{5}=0$, we have $\overline{\beta_{19}}=(0,0,1,0,0,1,0)$; 
c) when $\alpha_{3}=0, \alpha_{4} \neq 0, \alpha_{5}=0$, we have $\overline{\beta_{20}}=(0,0,0,1,0,1,0)$;

d) when $\alpha_{3}=0, \alpha_{4}=0, \alpha_{5} \neq 0$, we have $\overline{\beta_{21}}=(0,0,0,0, \pm 1,1,0)$;

e) when $\alpha_{3} \neq 0, \alpha_{4} \neq 0, \alpha_{5}=0$, we have $\overline{\beta_{22}}=(0,0,1,1,0,1,0)$;

f) when $\alpha_{3} \neq 0, \alpha_{4}=0, \alpha_{5} \neq 0$, we have $\overline{\beta_{23}}=(0,0,1,0, \pm 1,1,0)$;

g) when $\alpha_{3}=0, \alpha_{4} \neq 0, \quad \alpha_{5} \neq 0$, we have $\overline{\beta_{24}}=(0,0,0,1, \pm 1,1,0)$;

h) when $\alpha_{3}=0, \alpha_{4}=0, \alpha_{5}=0$, we have $\overline{\beta_{25}}=(0,0,0,0,0,1,0)$.

Case 2.3 When $\alpha_{7}=0, \alpha_{6}=0, \alpha_{5} \neq 0$, we obtain $\beta_{7}=0, \beta_{6}=0$. Equation (16) has the follow formula

$$
\frac{1}{a}\left(\begin{array}{c}
\alpha_{1} \\
\alpha_{2} \\
\alpha_{3} \mathrm{e}^{-2 \varepsilon_{2}}+2 \alpha_{2} \varepsilon_{3} \mathrm{e}^{-2 \varepsilon_{2}} \\
\mathrm{e}^{-\varepsilon_{2}}\left(\alpha_{4}+\alpha_{2} \varepsilon_{4}\right) \\
\alpha_{5} \mathrm{e}^{(1-\beta) \varepsilon_{1}-\varepsilon_{7}}+\alpha_{1} \varepsilon_{5}(-1+\beta) \mathrm{e}^{(1-\beta) \varepsilon_{1}} \\
0 \\
0
\end{array}\right)=\left(\begin{array}{c}
\beta_{1} \\
\beta_{2} \\
\beta_{3} \\
\beta_{4} \\
\beta_{5} \\
\beta_{6} \\
\beta_{7}
\end{array}\right) .
$$

We find that it can also be divided into four cases:

1) When $\alpha_{1} \neq 0, \alpha_{2} \neq 0$, let $\beta_{3}=\beta_{4}=\beta_{5}=0$, we can obtain $\varepsilon_{3}=-\frac{\alpha_{3}}{2 \alpha_{2}}$, $\varepsilon_{4}=-\frac{\alpha_{4}}{\alpha_{2}}, \quad \varepsilon_{5}=-\frac{\mathrm{e}^{-\varepsilon_{7}} \alpha_{5}}{\alpha_{1}(-1+\beta)}$, we have $\overline{\beta_{26}}=(\lambda, 1,0,0,0,0,0)$.

2) When $\alpha_{1} \neq 0, \alpha_{2}=0$, we find that it can also be divided four cases:

a) $\alpha_{3} \neq 0, \quad \alpha_{4} \neq 0$, we have $\overline{\beta_{27}}=(\lambda, 0,1,1,0,0,0)$;

b) $\alpha_{3} \neq 0, \alpha_{4}=0$, we have $\overline{\beta_{28}}=(1,0,1,0,0,0,0)$;

c) $\alpha_{3}=0, \alpha_{4} \neq 0$, we have $\overline{\beta_{29}}=(\lambda, 0,0,1,0,0,0)$;

d) $\alpha_{3}=0, \alpha_{4}=0$, we have $\overline{\beta_{30}}=(1,0,0,0,0,0,0)$.

3) When $\alpha_{1}=0, \alpha_{2} \neq 0$, we have $\overline{\beta_{31}}=(0,1,0,0, \pm 1,0,0)$.

4) When $\alpha_{1}=0, \alpha_{2}=0$, we find that it can also be divided four cases:

a) $\alpha_{3} \neq 0, \quad \alpha_{4} \neq 0$, we have $\overline{\beta_{32}}=(0,0,1,1, \pm 1,0,0)$;

b) $\alpha_{3} \neq 0, \quad \alpha_{4}=0$, we have $\overline{\beta_{33}}=(0,0,1,0, \pm 1,0,0)$;

c) $\alpha_{3}=0, \alpha_{4} \neq 0$, we have $\overline{\beta_{34}}=(0,0,0,1, \pm 1,0,0)$;

d) $\alpha_{3}=0, \alpha_{4}=0$, we have $\overline{\beta_{35}}=(0,0,0,0,1,0,0)$.

Case 2.4 When $\alpha_{7}=0, \alpha_{6}=0, \alpha_{5}=0, \alpha_{4} \neq 0$, we obtain $\beta_{7}=0, \beta_{6}=0$. Equation (16) has the follow formula

$$
\frac{1}{a}\left(\begin{array}{c}
\alpha_{1} \\
\alpha_{2} \\
\alpha_{3} \mathrm{e}^{-2 \varepsilon_{2}}+2 \alpha_{2} \varepsilon_{3} \mathrm{e}^{-2 \varepsilon_{2}} \\
\mathrm{e}^{-\varepsilon_{2}}\left(\alpha_{4}+\alpha_{2} \varepsilon_{4}\right) \\
\alpha_{1} \varepsilon_{5}(-1+\beta) \mathrm{e}^{(1-\beta) \varepsilon_{1}} \\
0 \\
0
\end{array}\right)=\left(\begin{array}{c}
\beta_{1} \\
\beta_{2} \\
\beta_{3} \\
\beta_{4} \\
\beta_{5} \\
\beta_{6} \\
\beta_{7}
\end{array}\right) .
$$

we find that it can also be divided into four cases: 
1) When $\alpha_{1} \neq 0, \alpha_{2} \neq 0$, let $\beta_{3}=0$, we can obtain $\varepsilon_{3}=-\frac{\alpha_{3}}{2 \alpha_{2}}, \varepsilon_{4}=-\frac{\alpha_{4}}{\alpha_{2}}$, we have $\overline{\beta_{36}}=(1, \lambda, 0,1, \pm 1,0,0)$.

2) When $\alpha_{1} \neq 0, \alpha_{2}=0$, if $\alpha_{3} \neq 0$, we have $\overline{\beta_{37}}=(\lambda, 0,1,1, \pm 1,0,0)$, if $\alpha_{3}=0$, we have $\overline{\beta_{38}}=(\lambda, 0,0,1, \pm 1,0,0)$.

3) When $\alpha_{1}=0, \alpha_{2} \neq 0$, let $\beta_{3}=0$, we obtain $\varepsilon_{3}=-\frac{\alpha_{3}}{2 \alpha_{2}} \quad \varepsilon_{4}=-\frac{\alpha_{4}}{\alpha_{2}}$, we have $\overline{\beta_{39}}=(0,1,0,1,0,0,0)$.

4) When $\alpha_{1}=0, \alpha_{2}=0$, if $\alpha_{3} \neq 0$, we have $\overline{\beta_{40}}=(0,0,1,1,0,0,0)$, if $\alpha_{3}=0$, we have $\overline{\beta_{41}}=(0,0,0,1,0,0,0)$.

Case 2.5 When $\alpha_{7}=0, \alpha_{6}=0, \alpha_{5}=0, \alpha_{4}=0, \alpha_{3} \neq 0$, we obtain $\beta_{7}=0$, $\beta_{6}=0$. Equation (16) has the follow formula

$$
\frac{1}{a}\left(\begin{array}{c}
\alpha_{1} \\
\alpha_{2} \\
\alpha_{3} \mathrm{e}^{-2 \varepsilon_{2}}+2 \alpha_{2} \varepsilon_{3} \mathrm{e}^{-2 \varepsilon_{2}} \\
\mathrm{e}^{-\varepsilon_{2}} \alpha_{2} \varepsilon_{4} \\
\alpha_{1} \varepsilon_{5}(-1+\beta) \mathrm{e}^{(1-\beta) \varepsilon_{1}} \\
0 \\
0
\end{array}\right)=\left(\begin{array}{c}
\beta_{1} \\
\beta_{2} \\
\beta_{3} \\
\beta_{4} \\
\beta_{5} \\
\beta_{6} \\
\beta_{7}
\end{array}\right) .
$$

We find that it can also be divided into four cases:

1) When $\alpha_{1} \neq 0, \alpha_{2} \neq 0$, we have $\overline{\beta_{42}}=(\lambda, 1,0,0, \pm 1,0,0)$;

2) When $\alpha_{1} \neq 0, \alpha_{2}=0$, we have $\overline{\beta_{43}}=(1,0,1,0, \pm 1,0,0)$;

3) When $\alpha_{1}=0, \alpha_{2} \neq 0$, we have $\overline{\beta_{44}}=(0,1,0,0,0,0,0)$;

4) When $\alpha_{1}=0, \alpha_{2}=0$, we have $\overline{\beta_{45}}=(0,0,1,0,0,0,0)$.

Case 2.6 When $\alpha_{7}=0, \alpha_{6}=0, \alpha_{5}=0, \alpha_{4}=0, \alpha_{3}=0, \alpha_{2} \neq 0$, Equation (16) has the follow formula

$$
\frac{1}{a}\left(\begin{array}{c}
\alpha_{1} \\
\alpha_{2} \\
2 \alpha_{2} \varepsilon_{3} \mathrm{e}^{-2 \varepsilon_{2}} \\
\mathrm{e}^{-\varepsilon_{2}} \alpha_{2} \varepsilon_{4} \\
\alpha_{1} \varepsilon_{5}(-1+\beta) \mathrm{e}^{(1-\beta) \varepsilon_{1}} \\
0 \\
0
\end{array}\right)=\left(\begin{array}{c}
\beta_{1} \\
\beta_{2} \\
\beta_{3} \\
\beta_{4} \\
\beta_{5} \\
\beta_{6} \\
\beta_{7}
\end{array}\right) .
$$

If $\alpha_{1} \neq 0$, we have $\overline{\beta_{46}}=(\lambda, 1,1,0, \pm 1,0,0) ;$ If $\alpha_{1}=0$, we have $\overline{\beta_{47}}=(0,1,1,0,0,0,0)$.

Case 2.7 When $\alpha_{7}=0, \alpha_{6}=0, \alpha_{5}=0, \alpha_{4}=0, \alpha_{3}=0, \alpha_{2}=0, \alpha_{1} \neq 0$, we have $\overline{\beta_{48}}=(1,0,0,0, \pm 1,0,0)$.

Following above calculate, where $\lambda$ is constants and $\lambda \neq 0$, we have found one-dimensional optimal system of Coupled Burgers' equations as follows:

$$
\begin{aligned}
& L_{1,1}=\left\{\lambda X_{1}+\lambda X_{2}+X_{7}\right\}, L_{1,2}=\left\{\lambda X_{1}+X_{3}+X_{4}+X_{7}\right\}, \\
& L_{1,3}=\left\{\lambda X_{1}+X_{3}+X_{7}\right\}, L_{1,4}=\left\{\lambda X_{1}+X_{4}+X_{7}\right\}, L_{1,5}=\left\{\lambda X_{1}+X_{7}\right\},
\end{aligned}
$$




$$
\begin{aligned}
& L_{1,6}=\left\{\lambda X_{2}+X_{7}\right\}, L_{1,7}=\left\{X_{3}+X_{4}+X_{7}\right\}, L_{1,8}=\left\{X_{3}+X_{7}\right\}, L_{1,9}=\left\{X_{4}+X_{7}\right\}, \\
& L_{1,10}=\left\{X_{7}\right\}, L_{1,11}=\left\{\lambda X_{1}+\lambda X_{2}+X_{6}\right\}, L_{1,12}=\left\{\lambda X_{1}+X_{3}+X_{4}+X_{6}\right\}, \\
& L_{1,13}=\left\{X_{1}+X_{3}+X_{6}\right\}, L_{1,14}=\left\{\lambda X_{1}+X_{4}+X_{6}\right\}, L_{1,15}=\left\{X_{1}+X_{6}\right\}, \\
& L_{1,16}=\left\{X_{2} \pm X_{5}+X_{6}\right\}, L_{1,17}=\left\{X_{2}+X_{6}\right\}, L_{1,18}=\left\{X_{3}+X_{4} \pm X_{5}+X_{6}\right\}, \\
& L_{1,19}=\left\{X_{3}+X_{6}\right\}, L_{1,20}=\left\{X_{4}+X_{6}\right\}, L_{1,21}=\left\{ \pm X_{5}+X_{6}\right\}, L_{1,22}=\left\{X_{3}+X_{4}+X_{6}\right\}, \\
& L_{1,23}=\left\{X_{3} \pm X_{5}+X_{6}\right\}, L_{1,24}=\left\{X_{4} \pm X_{5}+X_{6}\right\}, L_{1,25}=\left\{X_{6}\right\}, L_{1,26}=\left\{\lambda X_{1}+X_{2}\right\}, \\
& L_{1,27}=\left\{\lambda X_{1}+X_{3}+X_{4}\right\}, L_{1,28}=\left\{X_{1}+X_{3}\right\}, L_{1,29}=\left\{\lambda X_{1}+X_{4}\right\}, L_{1,30}=\left\{X_{1}\right\}, \\
& L_{1,31}=\left\{X_{2} \pm X_{5}\right\}, L_{1,32}=\left\{X_{3}+X_{4} \pm X_{5}\right\}, L_{1,33}=\left\{X_{3} \pm X_{5}\right\}, L_{1,34}=\left\{X_{4} \pm X_{5}\right\}, \\
& L_{1,35}=\left\{X_{5}\right\}, L_{1,36}=\left\{X_{1}+\lambda X_{2}+X_{4} \pm X_{5}\right\}, L_{1,37}=\left\{\lambda X_{1}+X_{3}+X_{4} \pm X_{5}\right\}, \\
& L_{1,38}=\left\{\lambda X_{1}+X_{4} \pm X_{5}\right\}, L_{1,39}=\left\{X_{2}+X_{4}\right\}, L_{1,40}=\left\{X_{3}+X_{4}\right\}, L_{1,41}=\left\{X_{4}\right\}, \\
& L_{1,42}=\left\{\lambda X_{1}+X_{2} \pm X_{5}\right\}, L_{1,43}=\left\{X_{1}+X_{3} \pm X_{5}\right\}, L_{1,44}=\left\{X_{2}\right\}, L_{1,45}=\left\{X_{3}\right\}, \\
& L_{1,46}=\left\{\lambda X_{1}+X_{2}+X_{3} \pm X_{5}\right\}, L_{1,47}=\left\{X_{2}+X_{3}\right\}, L_{1,48}=\left\{X_{1} \pm X_{5}\right\} .
\end{aligned}
$$

\subsection{Invariant Solutions of Coupled Burgers' Equations}

In this section, we only consider the Case 2 which is $\alpha=0, \beta \neq 0$. Let

$$
X_{8}=X_{3}+X_{4}+X_{5}=\frac{\partial}{\partial x}+\frac{\partial}{\partial t}+\left(u+\frac{v}{-1+\beta}\right) \mathrm{e}^{\psi+(-1+\beta) \phi} \frac{\partial}{\partial v}+\frac{\mathrm{e}^{\psi+(-1+\beta) \phi}}{-1+\beta} \frac{\partial}{\partial \psi} .
$$

The characteristic equations for the potential symmetry $X_{8}$ are as follows:

$$
\frac{\mathrm{d} x}{1}=\frac{\mathrm{d} t}{1}=\frac{\mathrm{d} u}{0}=\frac{\mathrm{d} v}{\left(u+\frac{v}{-1+\beta}\right) \mathrm{e}^{\psi+(-1+\beta) \phi}}=\frac{\mathrm{d} \phi}{0}=\frac{\mathrm{d} \psi}{\frac{\mathrm{e}^{\psi+(-1+\beta) \phi}}{-1+\beta}} .
$$

Solving Equations (23), we obtain the invariables $\theta=t-x$ and $u=c_{1}$, $\phi=c_{2}, \quad \psi=-\ln \left(\frac{\mathrm{e}^{-c_{2}+c_{2} \beta} x}{1-\beta}\right)-c_{3}, v=\frac{-c_{1} \mathrm{e}^{c_{2} \beta} x(-1+\beta)+c_{4}}{\mathrm{e}^{c_{2} \beta} x+c_{3} \mathrm{e}^{c_{2}}(-1+\beta)}$, where $c_{1}, c_{2}, c_{3}, c_{4}$ are arbitrary constants, we make $c_{1}=A_{1}(\theta), \quad c_{2}=A_{2}(\theta), \quad c_{3}=A_{3}(\theta)$, $c_{4}=A_{4}(\theta)$.

By using the invariant form method, we can confirm that the solutions of Equations (23) are given as follows:

$$
\left\{\begin{array}{l}
u=A_{1}(\theta) \\
v=\frac{-A_{1}(\theta) \mathrm{e}^{A_{2}(\theta) \beta} x(-1+\beta)+A_{4}(\theta)}{\mathrm{e}^{A_{2}(\theta) \beta} x+A_{3}(\theta) \mathrm{e}^{A_{2}(\theta)}(-1+\beta)} \\
\phi=A_{2}(\theta) \\
\psi=-\ln \left[\frac{\mathrm{e}^{A_{2}(\theta)+A_{2}(\theta) \beta} x}{1-\beta}-A_{3}(\theta)\right]
\end{array}\right.
$$

By substituting (24) into Equations (9), the system of ordinary differential equations we have got as follows: 


$$
\begin{gathered}
A_{1}(\theta)+A_{2}^{\prime}(\theta)=0, \\
A_{1}(\theta)^{2}+A_{1}^{\prime}(\theta)+A_{2}^{\prime}(\theta)=0, \\
\mathrm{e}^{\beta A_{2}(\theta)}+A_{4}(\theta)-\mathrm{e}^{A_{2}(\theta)}(-1+\beta) A_{3}^{\prime}(\theta)=0, \\
\mathrm{e}^{\beta A_{2}(\theta)}(-2+\beta) A_{2}^{\prime}(\theta)+\mathrm{e}^{A_{2}(\theta)}(-1+\beta)\left[\left(1+\beta A_{2}^{\prime}(\theta)\right) A_{3}^{\prime}(\theta)-A_{3}^{\prime \prime}(\theta)\right]=0 .
\end{gathered}
$$

We got the solutions of (25), (26) as follows:

$$
\left\{\begin{array}{l}
A_{1}(\theta)=\frac{\mathrm{e}^{\theta}}{\mathrm{e}^{\theta}-\mathrm{e}^{C_{1}}}, \\
A_{2}(\theta)=C_{2}-\ln \left(-\mathrm{e}^{\theta}+\mathrm{e}^{C_{1}}\right) .
\end{array}\right.
$$

We make $C_{1}=0$ and substitute $A_{1}(\theta), A_{2}(\theta)$ into the (27), (28), the solutions of $A_{1}(\theta), A_{2}(\theta), A_{3}(\theta), A_{4}(\theta)$ we have got as following

$$
\left\{\begin{aligned}
A_{3}(\theta) & =C_{4}+\frac{1}{(-1+\beta)^{2}} \mathrm{e}^{-C_{2}}\left[-\left(-1+\mathrm{e}^{\theta}\right)^{2}\right]^{-\beta}\left(-\mathrm{e}^{C_{2} \beta+\theta}\left(-1+\mathrm{e}^{\theta}\right)^{\beta}(-2+\beta) \theta\right. \\
& +\mathrm{e}^{C_{2} \beta}\left(\left(-1+\mathrm{e}^{\theta}\right)^{\beta}-\left(-\left(-1+\mathrm{e}^{\theta}\right)^{2}\right)^{\beta}\right)(-2+\beta) \theta-\mathrm{e}^{C_{2}+\theta}\left(1-\mathrm{e}^{\theta}\right)^{\beta} \\
& \times(-1+\beta) C_{3}-\mathrm{e}^{C_{2}}\left(1-\mathrm{e}^{\theta}\right)^{\beta}\left(-1+\left(1-\mathrm{e}^{\theta}\right)^{\beta}\right)(-1+\beta) C_{3}-\mathrm{e}^{C_{2} \beta+\theta} \\
& \left.\times\left(-\left(-1+\mathrm{e}^{\theta}\right)^{2}\right)^{\beta}\left(2-3 \beta+\beta^{2}\right) \text { HypergeometricPFQ}\left[(1,1, \beta),(2,2), \mathrm{e}^{\theta}\right]\right), \\
A_{4}(\theta) & =\left(1-\mathrm{e}^{\theta}\right)^{-1-\beta}\left(-\left(-1+\mathrm{e}^{\theta}\right)^{2}\right)^{-\beta}\left(C_{3} \mathrm{e}^{C_{2}+\theta}\left(1-\mathrm{e}^{\theta}\right)^{2 \beta}(-1+\beta)\right. \\
& \left.+\mathrm{e}^{C_{2} \beta}\left(-\left(-1+\mathrm{e}^{\theta}\right)^{2}\right)^{\beta}\left(-1+\mathrm{e}^{\theta}(1+(-2+\beta) \theta)\right)\right) .
\end{aligned}\right.
$$

where $C_{2}, C_{3}, C_{4}$ are arbitrary constants and HypergeometricPFQ is the generalized hypergeometric function. By substituting (29) (30) into (24), we confirm the invariant solutions of Equations (7) (8) are given as follows:

$$
\begin{gathered}
u(x, t)=\frac{1}{1-\mathrm{e}^{-t+x}}, \\
v(x, t)=\frac{-B_{1}^{-\beta}\left(-B_{1}^{2}\right)^{-\beta}\left[\mathrm{e}^{C_{2} \beta}\left(-\mathrm{e}^{-2 x} B_{2}^{2}\right)^{\beta} B_{3}+C_{3} \mathrm{e}^{C_{2}+t} B_{1}^{2 \beta}(-1+\beta)\right]}{B_{2}\left[B_{8} B_{9}\left(B_{1}^{-\beta} B_{9}^{-1} B_{7}-C_{3} \mathrm{e}^{C_{2}} B_{1}^{\beta} B_{6}(-1+\beta)\right)+B_{12}\right]},
\end{gathered}
$$

where $B_{13}=$ HypergeometricPFQ $\left[(1,1, \beta),(2,2), \mathrm{e}^{t-x}\right], B_{1}, B_{2}, B_{3}, B_{4}, B_{5}$, $B_{6}, B_{7}, B_{8}, B_{9}, B_{10}, B_{11}, B_{12}$ have the following expressions:

$$
\begin{gathered}
B_{1}=1-\mathrm{e}^{t-x}, B_{2}=\mathrm{e}^{t}-\mathrm{e}^{x}, B_{3}=-\mathrm{e}^{x}+\mathrm{e}^{t}(1+x+t(-2+\beta)), \\
B_{4}=-\mathrm{e}^{t}(x+t(-2+\beta)), B_{5}=\mathrm{e}^{x}\left(x+\left(t+B_{1}^{\beta}(-t+x)\right)(-2+\beta)\right), \\
B_{6}=\mathrm{e}^{t}+\mathrm{e}^{x}\left(-1+\left(1-\mathrm{e}^{t-x}\right)^{\beta}\right), B_{7}=B_{10}+C_{4} B_{11}, B_{8}=\frac{1}{B_{2}(-1+\beta)}, \\
B_{9}=\left(-\mathrm{e}^{-2 x} B_{2}^{2}\right)^{-\beta}, B_{10}=\mathrm{e}^{C_{2} \beta}\left(B_{4}+B_{5}\right),
\end{gathered}
$$




$$
B_{11}=\mathrm{e}^{C_{2}+x} B_{1}^{\beta}(-1+\beta)^{2}, B_{12}=\frac{\mathrm{e}^{t+C_{2} \beta}(-2+\beta) B_{13}}{\mathrm{e}^{t}-\mathrm{e}^{x}} .
$$

It is important that these solutions cannot be obtained by the classical symmetries of Coupled Burgers' equations. In this paper, we only consider $\beta=2$ which the reason is that the form of solutions (31) (32) are too complex, and confirm the new solutions of Equations (7) (8) are given as follows:

$$
\begin{gathered}
u(x, t)=\frac{1}{1-\mathrm{e}^{-t+x}}, \\
v(x, t)=\frac{\mathrm{e}^{x}\left[C_{3} \mathrm{e}^{t}+\mathrm{e}^{C_{2}}\left(\mathrm{e}^{t}-\mathrm{e}^{x}+x \mathrm{e}^{t}\right)\right]}{\left(-\mathrm{e}^{t}+\mathrm{e}^{x}\right)\left(C_{3} \mathrm{e}^{t}+C_{4}\left(-\mathrm{e}^{t}+\mathrm{e}^{x}\right)+\mathrm{e}^{C_{2}+x} x\right)} .
\end{gathered}
$$

The Lie group method is probably the most powerful method to find the exact solutions of nonlinear PDEs. A symmetry group of PDEs is a group of transformations which maps any solution to another solution of PDEs. For obtaining more exact solutions of Coupled Burgers' equations. Corresponding to the symmetry, Lie transformation group is determined. A one-parameter Lie transformation group corresponding to the symmetry $X_{9}=X_{1}+X_{2}+X_{3}+X_{5}$ satisfies the initial value problem

$$
(I)\left\{\begin{array}{l}
x^{*}=\mathrm{e}^{-\varepsilon} x, t^{*}=\frac{1}{2}\left(1+\mathrm{e}^{-2 \varepsilon}(-1+2 t)\right), u^{*}=\mathrm{e}^{\varepsilon} u, \phi^{*}=\varepsilon+\phi, \\
v^{*}=\frac{\left(-\mathrm{e}^{\varepsilon+\beta \phi+\psi} u+\mathrm{e}^{\beta(\varepsilon+\phi)+\psi} u+\mathrm{e}^{\varepsilon+\phi} v(-1+\beta)\right)(-1+\beta)}{\mathrm{e}^{\beta \phi+\psi}-\mathrm{e}^{(-1+\beta) \varepsilon+\beta \phi+\psi}+\mathrm{e}^{\phi}(-1+\beta)^{2}}, \\
\psi^{*}=-\ln \left(\frac{\mathrm{e}^{-\phi-\psi}\left(\mathrm{e}^{\beta \phi+\psi}-\mathrm{e}^{(-1+\beta) \varepsilon+\beta \phi+\psi}+\mathrm{e}^{\phi}(-1+\beta)^{2}\right)}{(-1+\beta)^{2}}\right) .
\end{array}\right.
$$

By acting Lie transformation group (I) on the solution (33) (34) of Equations (7) (8), we obtain another solutions of Equations (7) (8).

$$
\begin{gathered}
u_{1}(x, t)=\frac{\mathrm{e}^{\varepsilon+\frac{1}{2}\left(1-\mathrm{e}^{2 \varepsilon+2 \mathrm{e}^{2 \varepsilon t}}\right)}}{\mathrm{e}^{\frac{1}{2}\left(1-\mathrm{e}^{2 \varepsilon}+2 \mathrm{e}^{2 \varepsilon t}\right)}-\mathrm{e}^{\mathrm{e}^{\varepsilon} x}}, \\
v_{1}(x, t)=\frac{\mathrm{e}^{C_{2}+\mathrm{e}^{2 \varepsilon}+\varepsilon+2 \mathrm{e}^{\varepsilon} x}-D_{0}\left(C_{3}+\mathrm{e}^{C_{2}+\varepsilon}(1+x)\right)}{\left(\mathrm{e}^{\frac{1}{2}+\mathrm{e}^{2 \varepsilon} t}-\mathrm{e}^{\frac{1}{2} \varepsilon^{\varepsilon}\left(\mathrm{e}^{\varepsilon}+2 x\right)}\right)\left(D_{1}+D_{2}\left(-1+\mathrm{e}^{\varepsilon}(-1+x)\right)\right)} .
\end{gathered}
$$

where $D_{0}=\mathrm{e}^{\frac{1}{2}+\mathrm{e}^{2 \varepsilon}+\varepsilon+\mathrm{e}^{2 \varepsilon} t+\mathrm{e}^{\varepsilon} x}, \quad D_{1}=C_{3} \mathrm{e}^{\frac{1}{2}+\mathrm{e}^{2 \varepsilon} t}-C_{4} \mathrm{e}^{\frac{1}{2}+\mathrm{e}^{2 \varepsilon} t}+C_{4} \mathrm{e}^{\frac{1}{2} \mathrm{e}^{\varepsilon}\left(\mathrm{e}^{\varepsilon}+2 x\right)}$, $D_{2}=\mathrm{e}^{C_{2}+\frac{\mathrm{e}^{2 \varepsilon}}{2}+\mathrm{e}^{\varepsilon} x}$.

In addition, we can also continue the above calculation by acting Lie transformation groups and obtain more new solutions. Due to the lack of space, we omit the calculation steps. 


\section{Conclusions}

In this paper, we expanded the classical symmetries of Coupled Burgers' equations by the determining potential symmetries, and we obtained new exact solutions of Coupled Burgers' equations. Firstly, we determined the classical symmetries and the potential symmetry of Coupled Burgers' equations based on differential characteristic set algorithm. Secondly, we determined one-dimensional optimal system of Coupled Burgers' equations. Finally, we obtained the invariant solutions of the potential symmetry and one-parameter Lie transformation groups of the potential symmetries. Moreover the exact solutions of Coupled Burgers' equations are derived by applying Lie transformation groups (I). It is important that these solutions cannot be obtained from the classical symmetries of Coupled Burgers' equations. These solutions are different from those solutions given in literature [17].

We can also obtain the exact solutions of Coupled Burgers' equations by using the modern algebraically methods, such as the tanh-function method [19], the simplest equation method [20] etc. After that we construct new exact solutions by acting Lie transformation group of the potential symmetry on these exact solutions. Due to the lack of space, we omit the other calculation.

\section{Acknowledgements}

This work is supported by National Natural Science Foundation of China (11661060) (11571008). Natural Science Foundation of Inner Mongolia Autonomous Region of China (2018LH01013).

\section{Conflicts of Interest}

The authors declare no conflicts of interest regarding the publication of this paper.

\section{References}

[1] Bluman, G.W. and Kumei, S. (1989) Symmetries and Differential Equations. Spring-Verlag, Berlin. https://doi.org/10.1007/978-1-4757-4307-4

[2] Bluman, G.W., Cheviakov, A. and Anco, S. (2010) Applications of Symmetry Methods to Partial Differential Equations. Spring-Verlag, New York. https://doi.org/10.1007/978-0-387-68028-6

[3] Olver, P.J. (1986) Applications of Lie Groups to Differential Equations. Spinger-Verlag, New York. https://doi.org/10.1007/978-1-4684-0274-2

[4] Gaur, M. and Singh, K. (2017) Symmetry Analysis of Time-Fractional Potential Burgers' Equation. Mathematical Communications, 22, 1-11.

[5] Ma, W.X. (2013) A Soliton Hierarchy Associated with so(3,R). Applied Mathematics and Computation, 220, 117-122. https://doi.org/10.1016/j.amc.2013.04.062

[6] Bluman, G.W. and Chaolu, T. (2005) Conservation Laws of Nonlinear Telegraph Equations. Journal of Mathematical Analysis and Applications, 310, 459-476. https://doi.org/10.1016/j.jmaa.2005.02.007

[7] Motsepa, T., Khalique, C. and Gandarias, M. (2017) Symmetry Analysis and Con- 
servation Laws of the Zoomeron Equation. Symmetry, 9, 27. https://doi.org/10.3390/sym9020027

[8] Ma, W.X. (2015) Conservation Laws of Discrete Evolution Equations by Symmetries and Adjoint Symmetries. Symmetry, 7, 714-725.

https://doi.org/10.3390/sym7020714

[9] Wang, G.W., Liu, X.Q. and Zhang, Y.Y. (2013) Symmetry Reduction, Exact Solutions and Conservation Laws of a New Fifth Order Nonlinear Integrable Equation. Communications in Nonlinear Science and Numerical Simulation, 18, 2313-2320. https://doi.org/10.1016/j.cnsns.2012.12.003

[10] Eerdun, B.H. and Bluman, G.W. (2015) Symmetry Reductions, Exact Solutions, and Conservation Laws of the Generalized Zakharov Equations. Journal of Mathematical Physics, 56, Article ID: 101501.

[11] Gai, L.T., Bilige, S.D. and Jie, Y.M. (2016) The Exact Solutions and Approximate Analytic Solutions of (2+1)-Dimensional KP Equation Based on Symmetry Method. SpringerPlus, 5, 1267. https://doi.org/10.1186/s40064-016-2908-8

[12] Bilige; S.D., Wang, X.M. and Morigen, W.Y. (2014) Application of the Symmetry Classification to the Boundary Value Problem of Nonlinear Partial Differential Equations. Acta Physica Sinica, 4, 6-12. (In Chinese)

[13] Bluman, G.W., Reid, G.J. and Kumei, S. (1998) New Classes of Symmetries for Partial Differential Equations. Journal of Mathematical Physics, 29, 806-811. https://doi.org/10.1063/1.527974

[14] Popovych, R.O., Kunzinger, M. and Ivanova, N.M. (2008) Conservation Laws and Potential Symmetries of Linear Parabolic Equations. Acta Applicandae Mathematicae, 100, 113-185. https://doi.org/10.1007/s10440-007-9178-y

[15] Huang, Q., Qu, C.Z. and Zhdanov, R. (2011) Group-Theoretical Framework for Potential Symmetries of Evolution Equations. Journal of Mathematical Physics, 52, Article ID: 023514. https://doi.org/10.1063/1.3554692

[16] Sophocleous, C. and Wiltshire, R.J. (2006) Linearisation and Potential Symmetries of Certain Systems of Diffusion Equations. Physica A, 370, 329-345. https://doi.org/10.1016/j.physa.2006.03.003

[17] Seadawy, A. and El-Rashidy, R.K. (2015) Classification of Multiply Travelling Wave Solutions for Coupled Burgers, Combined KdV-Modified KdV, and Schrödinger-KdV Equations. Abstract and Applied Analysis, 2015, Article ID: 369294. https://doi.org/10.1155/2015/369294

[18] Chaolu, T. and Bai, Y.S. (2010) A New Algorithmic Theory for Determining and Classifying Classical and Non-Classical Symmetries of Partial Differential Equations. Scientia Sinica, 40, 331-348. (In Chinese)

[19] Soliman, A.A. (2006) The Modified Extended Tanh-Function Method for Solving Burgers-Type Equations. Physica A, 361, 394-404. https://doi.org/10.1016/j.physa.2005.07.008

[20] Bilige, S.D. and Wang, X.M. (2015) A Generalized Simplest Equation Method and Its Application to the Boussinesq-Burgers Equation. PLoS ONE, 10, e0126635. 